\title{
Long-term studies of the Cygnus Region and its objects
}

\section{V.Y. Sinitsyna*}

P.N. Lebedev Physical Institute, Leninsky pr. 53, Moscow, Russia

E-mail: sinits@sci.lebedev.ru

\section{V.G. Sinitsyna}

P.N. Lebedev Physical Institute, Leninsky pr. 53, Moscow, Russia

E-mail: sinits@sci.lebedev.ru

The nineteen-year-long studies of the Cygnus Region at energies $800 \mathrm{GeV}-100 \mathrm{TeV}$ by SHALON telescope are presented in this paper. The long-term observations of the Cygnus region centered on Cyg X-3 with the SHALON telescope are revealed the $\gamma$-ray emission from the one of nearby object - $\gamma$ Cygni SNR, placed at $\sim 2^{o}$ from Cyg X-3. The results of $\gamma$ Cygni SNR observation since $1995 \mathrm{y}$ by SHALON are presented with spectral energy distribution, images and integral spectra at energies $800 \mathrm{GeV}-50 \mathrm{TeV}$. The results of nineteen-year observations of the Cyg X-3 binary at energies $800 \mathrm{GeV}-85 \mathrm{TeV}$, detected by SHALON in 1995y are presented with images and integral spectra. A number of high activity period of Cyg X-3 were detected at energies $>800 \mathrm{GeV}$ during the all observation time. The correlation soft X-ray and TeV energy $\gamma$-ray fluxes is traced.

Frontiers of Fundamental Physics 14 - FFP14,

15-18 July 2014

Aix Marseille University (AMU) Saint-Charles Campus, Marseille

\footnotetext{
* Speaker.
} 


\section{Introduction}

SHALON is a high-altitude imaging atmospheric Cherenkov telescope for the detection of very high energy (from $800 \mathrm{GeV}$ to $100 \mathrm{TeV}$ ) $\gamma$-rays. The $\gamma$-astronomical researches are carrying out with SHALON telescope since 1992. During the period 1992 - 2014 SHALON has been used for observations of different galactic and extragalactic objects (see table 1 and [1]).

The SHALON observations have yielded the results on different Galactic sources, such as supernova remnants (SNR) of different types and ages, binaries, etc. Among them are: the shell-type SNRs Tycho's SNR, Cas A, IC 443, $\gamma$ Cygni SNR and the plerions Crab Nebula, 3C58, Geminga (probably plerion) [1]. For each of sources the observation results are presented with spectral energy distribution by SHALON in comparison with other experiment data and images by SHALON in comparison with data from X-ray and radio-data by CGPS (for $\gamma$ Cygni SNR).

Cygnus Region contains a number of sources of radio and X-ray emission and some of them were detected at high energies. Among them are Cyg X-3 and the nearby object - $\gamma$ Cygni SNR.

\section{Cygnus X-3}

Binary system Cyg X-3 is one of the brightest Galactic X-ray sources, displaying high and low states and rapid variability in X-rays. It is also the strongest radio source among X-ray binaries and shows both huge radio outbursts and relativistic jets. The radioactivity is closely linked with the $\mathrm{X}$-ray emission and the different X-ray states $[2,3]$. Based on the detections of ultra high energy $\gamma$-rays [4, 5], Cygnus X-3 has been proposed to be one of the most powerful sources of charged cosmic ray particles in the Galaxy (Fig. 1).

Cyg X-3 has been regularly observed since a 1995 with SHALON telescope [6] during the 283.6 hours in total. The $\gamma$-ray source associated with the Cyg X-3 was detected above $800 \mathrm{GeV}$ with a statistical significance [7] of $35.3 \sigma$ with a average integral $\gamma$-ray flux above $800 \mathrm{GeV} F\left(E_{O}>\right.$ $0.8 \mathrm{eV})=(6.8 \pm 0.4) \times 10^{-13} \mathrm{~cm}^{-2} \mathrm{~s}^{-1}[8,9,10,11]$. The energy spectrum of Cyg X-3 at $800 \mathrm{GeV}$ - $85 \mathrm{TeV}$ can be approximated by the power law $F\left(>E_{O}\right) \propto E^{-1.25 \pm 0.10}$.

Table 1: The catalogue of galactic $\gamma$-ray sources by SHALON with parameters for spectrum fitting in form of power low with exponential cutoff $F(>E) \propto E^{k_{\gamma}} \times \exp \left(-E / E_{\text {cutoff }}\right)$.

\begin{tabular}{cccccc}
\hline Sources & Observable flux $^{a}$ & $k_{\gamma}$ & $E_{\text {cutoff }}, \mathrm{TeV}$ & Distance, kpc & Type \\
\hline Crab Nebula & $(2.12 \pm 0.12)$ & $-1.36 \pm 0.09$ & $19.0 \pm 2.0$ & 2.0 & PWN \\
Geminga & $(0.48 \pm 0.07)$ & $-0.39 \pm 0.05$ & $5.4 \pm 1.0$ & 0.25 & PSR or PWN \\
3C 58 & $(0.56 \pm 0.15)$ & $-1.33 \pm 0.12$ & - & 3.2 & PWN \\
Tycho's SNR & $(0.52 \pm 0.04)$ & $-0.93 \pm 0.09$ & $35.0 \pm 5.0$ & $3.1-3.3$ & Shell-type SNR \\
Cas A & $(0.64 \pm 0.10)$ & $-0.91 \pm 0.11$ & $10.3 \pm 2.5$ & 3.1 & Shell-type SNR \\
IC 443 & $(1.69 \pm 0.58)$ & $-1.94 \pm 0.16$ & - & 1.5 & Shell-type SNR \\
rCygni SNR & $(1.27 \pm 0.11)$ & $-0.95 \pm 0.09$ & $20.1 \pm 4.2$ & 1.5 & Shell-type SNR \\
GK Per & $(0.31 \pm 0.14)$ & $-1.90 \pm 0.36$ & - & 0.46 & Classical Nova \\
Cyg X-3 & $(0.68 \pm 0.04)$ & $-1.15 \pm 0.08$ & $75.0 \pm 10.2$ & 10.0 & HMX Binary \\
4U 2129+47 & $(0.19 \pm 0.06)$ & $-0.42 \pm 0.12$ & $10.0 \pm 3.0$ & 6.0 & LMX Binary \\
Her X-1 & $(0.45 \pm 0.18)$ & - & - & 6.6 & Binary \\
M57 & $(0.30 \pm 0.17)$ & - & - & 0.7 & Planetary nebula \\
\hline \multicolumn{7}{c}{ Integral flux at energy $>800 \mathrm{GeV}$ in units of $10^{-12} \mathrm{~cm}^{-2} \mathrm{~s}^{-1}$} &
\end{tabular}




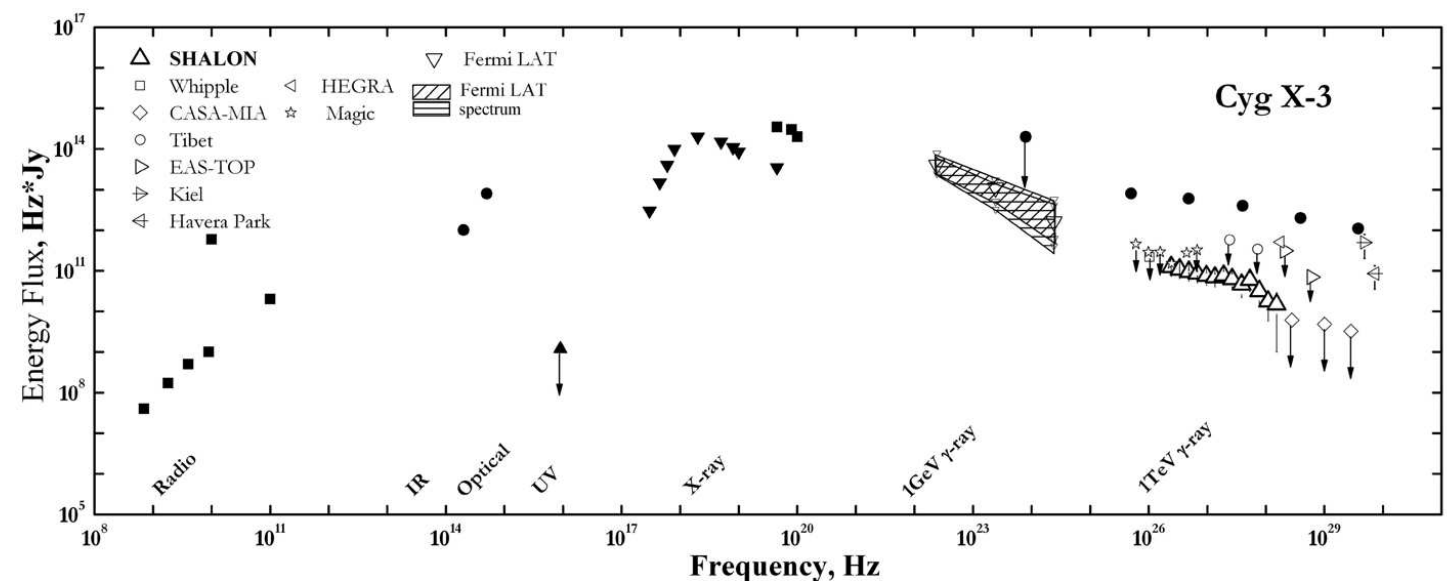

Figure 1: Spectral energy distribution of the $\gamma$-ray emission from Cyg X-3. $\triangle$ represent the data from the SHALON ground-based Cherenkov telescope. Black points are the archival data from [12].
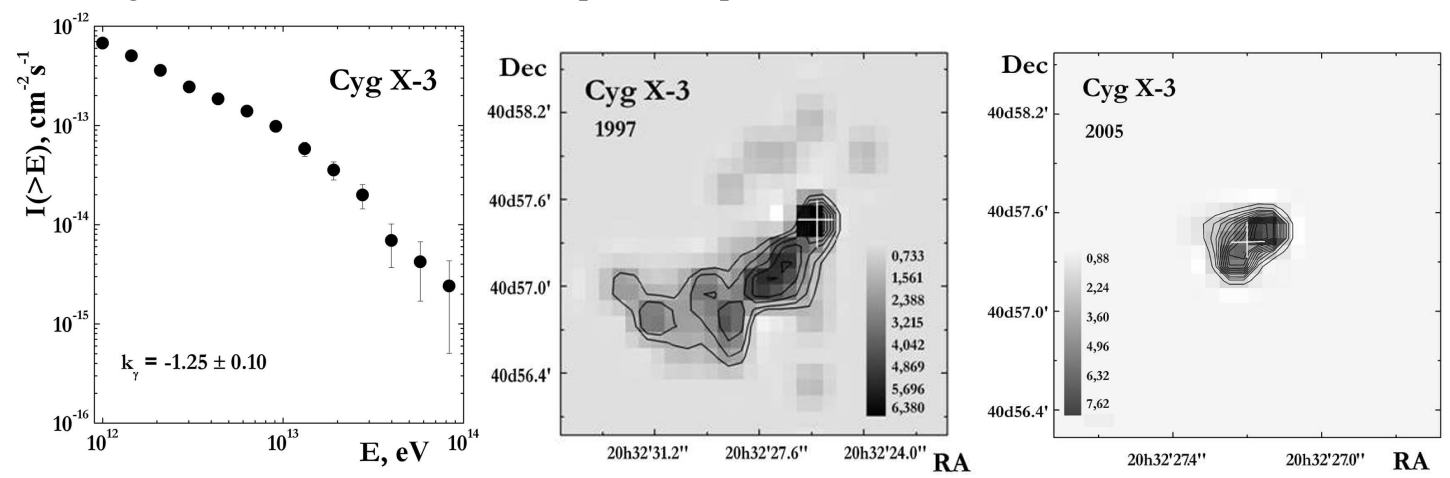

Figure 2: from left to right:The integral energy spectrum of Cyg X-3; the images of Cyg X-3 in flaring period of $1997 \mathrm{y}$ and in the silent period of $2005 \mathrm{y}$ by SHALON

Extreme variability in different wavelengths including VHE $\gamma$-rays is the remarkable feature of Cyg X-3. A number of high activity period of Cyg X-3 were detected with SHALON at energies $>800 \mathrm{GeV}$ during the all period of observation since $1995 \mathrm{y}$. For example, the images of Cyg X-3 in silent period at 2005 and flaring period of 1997y are shown in comparison (Fig. 2). There are no features revealed at flaring periods found at 2005y. The last two significant increase of very high energy $\gamma$-ray flux have detected in May 2009 and October 2011, which is correlated with flaring activity at lower energy range of soft X-ray and/or at observations of Fermi LAT[13]. Also, the high TeV $\gamma$-ray flux was detected by SHALON during the X-ray flares of end September and mid of October 2014 observed by MAXI [14]. Earlier, in 1997, 2003 and 2006 a comparable increase of the flux over the average value was also observed.

During the period of observations of Cyg X-3 with SHALON 6 significant flux increases were detected at energies above $0.8 \mathrm{TeV}$. To reveal possible correlation of periods of activity in the TeV energy range with the flares at the low energies the light curves of Swift/BAT $(15-50 \mathrm{keV}){ }^{1}$, MAXI $(2-4 \mathrm{keV})$ [14], RXTE/ASM $(3-5 \mathrm{keV})^{2}$, the fluxes at radio-ranges from RATAN (11.2GHz)(see [15]), AMI-LA (15 GHz) ${ }^{3}$ and TeV fluxes from SHALON observations were analyzed (Fig. 3).

\footnotetext{
${ }^{1}$ http://swift.gsfc.nasa.gov/results/transients/CygX-3/

${ }^{2} \mathrm{http}: / / \mathrm{xte}$.mit.edu/asmlc/srcs/cygx3.html

${ }^{3}$ http://www.mrao.cam.ac.uk/ guy/cx3/
} 


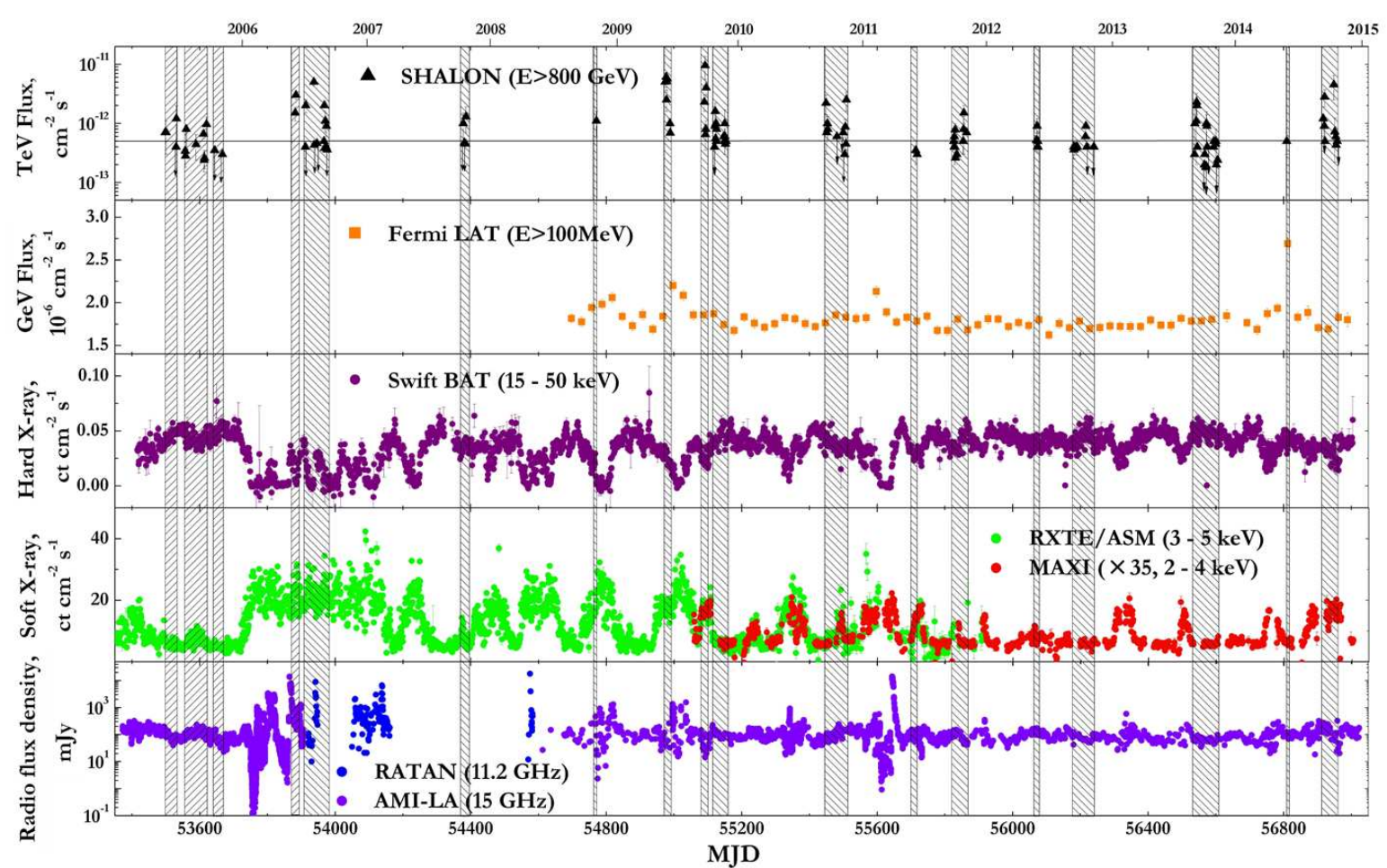

Figure 3: The light curves of Cyg X-3 in wide energy range.

The significant anticorrelation of the fluxes at $\mathrm{TeV}$ and hard $\mathrm{X}$-rays and the correlation of very high energy flux and soft X-ray were found. It is note, that $\mathrm{TeV}$ flaring activities occur within the 4 - 5 days to strong radio flares. Probably, it is linked with the powerful ejection from the regions are close to the centers blackhole. This ejection is accompanied with a relativistic shock where the relativistic electrons and magnetic field are generated effectively. Similar relation of TeV and soft X-ray fluxes were found in the 1997 observation period. But the flux increase of 2003 didn't obey this scheme, it was in the quite period in the soft X-rays. In general, the correlation soft X-ray and $\mathrm{TeV}$ energy $\gamma$-ray fluxes is traced since 1996y.

\section{$\gamma$ Cygni SNR}

Supernova remnant G78.2+2.1, known as $\gamma$ Cygni SNR is a shell-type supernova remnant at a distance of $\sim 1-2 \mathrm{kpc}$ and with the observed diameter of $\sim 1^{\circ}$. The shell-like features are known in radio- and X-ray energy regions. YCygni SNR is older then Cas A and Tycho's SNR, its age is estimated as $\sim 5000-7000$ yr. [16, 17] and its supposed to be and in an early phase of adiabatic expansion. The observations of different age supernova remnants can help to reveal the mechanisms of very high energy cosmic ray acceleration in the SNRs.

During the observations of Cyg X-3 the SHALON field of view contains $\gamma$ Cygni SNR as it located in Cygnus Region at $\sim 2^{\circ} \mathrm{SW}$ from Cyg X-3. So due to the large telescopic field of view $\left(\geq 8^{\circ}\right)$ the observations of Cyg X-3 is naturally followed by the tracing of $\gamma$ Cygni SNR.

$\gamma$ Cygni SNR as a source accompanying to Cyg X-3 is systematically studied with SHALON telescope since 1995y. $\gamma$ Cygni SNR was observed with SHALON telescope during the period from $1995 y$ till now for a total of 249.8 hours. The $\gamma$-ray source associated with the $\gamma$ Cygni SNR was detected above $800 \mathrm{GeV}$ with average $\gamma$-flux above $0.8 \mathrm{TeV}(1.27 \pm 0,11) \times 10^{-12} \mathrm{~cm}^{-2} \mathrm{~s}^{-1}$ [18]. The energy spectrum of $\gamma$-rays in the energy region from $800 \mathrm{GeV}$ to $50 \mathrm{Tev}$ is well described by the 

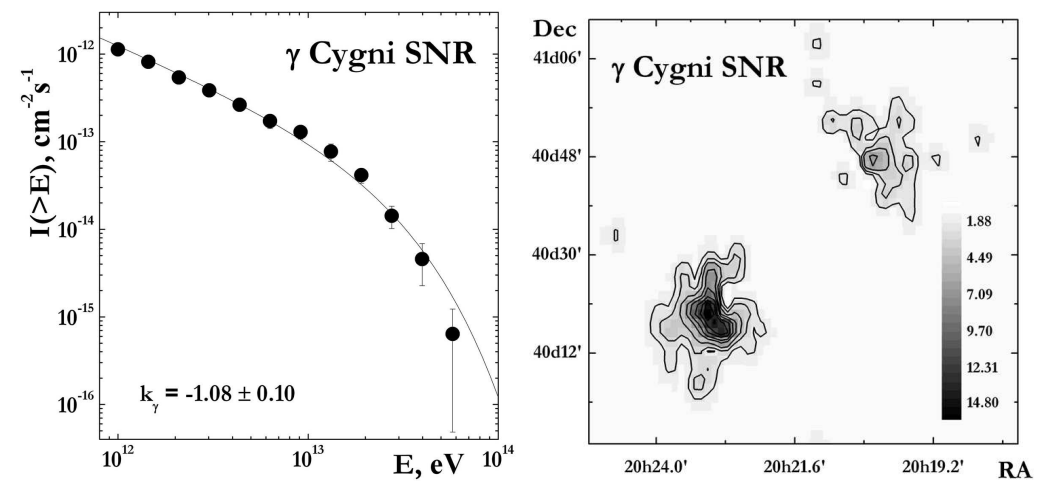

Figure 4: left: The integral energy spectra for $\gamma$ Cygni SNR; right: Image of $\gamma$ Cygni SNR at energies $>800 \mathrm{GeV}$ measured by SHALON
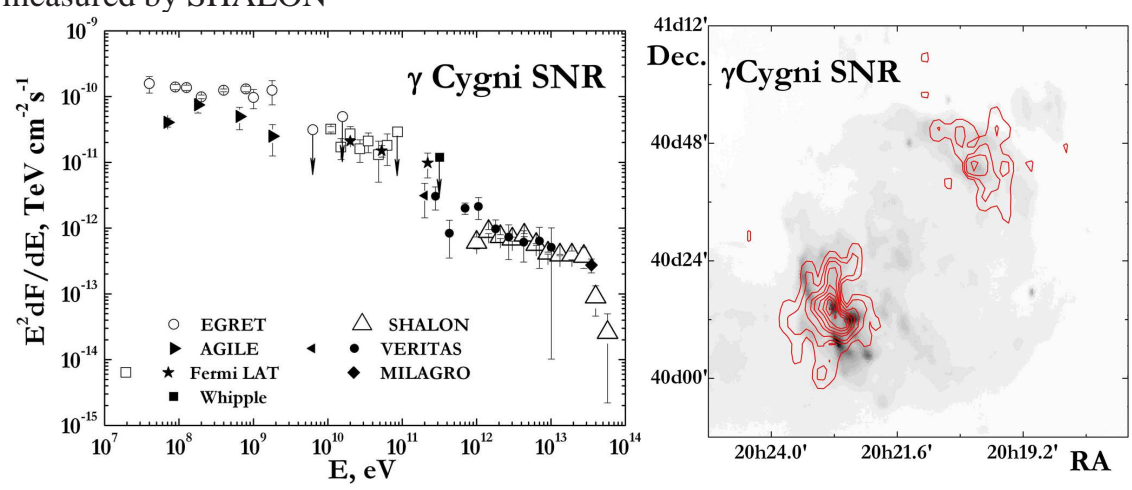

Figure 5: left: The spectral energy distribution of the $\gamma$-ray emission from $\gamma$ Cygni SNR by SHALON in comparison with other experiment data; right: The comparison of $\gamma$ Cygni SNR images in radio by CGPS and $\mathrm{TeV}$ energies by SHALON (red lines)

power law with exponential cutoff, $I\left(>E_{\gamma} / 1 \mathrm{TeV}\right)=(1.25 \pm 0.10) \times 10^{-12} \times\left(E_{\gamma} / 1 \mathrm{TeV}\right)^{-0.95 \pm 0.09} \times$ $\exp \left(-E_{\gamma} / 20 \mathrm{TeV}\right) \mathrm{cm}^{-2} \mathrm{~s}^{-1}$ (Fig. 4, left). Also, the $\gamma$-ray integral spectrum of $\gamma$ Cygni SNR at energies $0.8-28 \mathrm{TeV}$ can be approximated with a power law: $I\left(>E_{\gamma}\right) \propto E^{-1.08 \pm 0.10}$. The image of $\gamma$ Cygni SNR by SHALON are shown with Fig. 4 , right. The $\gamma$-ray source associated with the $\gamma$ Cygni SNR was detected above $800 \mathrm{GeV}$ with a statistical significance[7] of $19.5 \sigma$. The signal significance for this SNR is less then one for the source with similar flux and spectrum index obtained in the same observation hours because of less collection field of view relative to the standard procedure of SHALON experiment. The corrections for the effective field of view were made to calculate source flux and energy spectrum. Taking into account the proximity to a nearby source Cyg X-3, we made the observation data procession first associated with Cyg X-3 and then with YCygni SNR. We found that $2.4 \%$ of showers are common for the both sources. After the detailed analysis of arrival direction of these showers and angular distance less than $1 \%$ of Cyg X-3 showers were recognized to be SNR showers. This didn't change the average flux of Cyg X-3.

The spectral energy distribution of the $\gamma$-ray emission from $\gamma$ Cygni SNR by SHALON [18] is presented in comparison with experiment data from Fermi LAT[19](2009 - 2011), EGRET[20, 21] (1995, 1996), AGILE[22](2010), VERITAS[23](2013), MILAGRO[24](2011) (Fig. 5, left). (Left triangle symbol at $200 \mathrm{GeV}$ in Fig. 5 is flux of VERJ2019+407 by VERITAS [25] (2009)).

The comparison of images in radio by CGPS and TeV energies by SHALON (red lines) is presented with Fig. 5, right. TeV $\gamma$-ray emission regions correlate with the NW and SE parts of the shell visible in the radio energies by CGPS (the Canadian Galactic Plane Survey). 


\section{Conclusion}

Cygnus Region contains the number of powerful sources of radio and X-rays which are supposed as a potential TeV-emitting objects. The results of 19-year observations of the Cyg X-3 at energies $0.8-85 \mathrm{TeV}$, detected by the SHALON telescope in 1995 are presented. A number of high activity period of Cyg X-3 were detected with SHALON during the all period of observation. The significant increases of flux are correlated with flaring activity at lower energy range of X-ray and/or at observations of Fermi LAT. Also, long-term observations of the Cygnus region are revealed the $\gamma$-ray emission from the one of nearby object $-\gamma$ Cygni SNR, placed at $2^{\circ}$ from Cyg X-3. The results of $\gamma$ Cygni SNR observation by SHALON are presented with spectral energy distribution, images and integral spectra at energies $0.8-50 \mathrm{TeV}$. The correlation of $\mathrm{TeV} \gamma$-ray emission regions and the shell visible in the radio energies by CGPS is found.

\section{References}

[1] V. G. Sinitsyna and V. Yu. Sinitsyna, Astron. Lett. 37(9), 621 (2011).

[2] M. L. Mccollough et al. Astrophys. J. 517, 951 (1999)

[3] S. A. Trushkin, et al. Proc. of IAU Symposium No. 238, 2006 eds. V. Karas, G. Matt, 2007, p. 463.

[4] M. Samorscki and W. Stamm, Astrophys. J., 268, L17 (1983).

[5] J. Lloyd-Evans et al., Nature, 305, 784 (1983).

[6] V. G. Sinitsyna, Nuovo Cim. 19C, 965 (1996).

[7] T.-P. Li and Y.-Q. Ma, Astrophys. J. 272, 317 (1983)

[8] V. G. Sinitsyna, AIP, 515, 205, 293 (2000).

[9] V. G. Sinitsyna et al., Int. J. Mod. Phys. A, 29, 7023 (2005).

[10] V. G. Sinitsyna Rad. Phys. and Chem., 75, 880 (2006).

[11] V. G. Sinitsyna et al., J. Phys. Soc. Jpn., Suppl. A, 78, 92 (2009).

[12] F. A.-D. Cordova, Los Alamos Science, Spring, 39 (1986).

[13] A. A. Abdo et al. Science, 326, 1512 (2009).

[14] M. Matsuoka, et al., PASJ, 61, 999 (2009)

[15] J. Alekśic et al. ApJ, 721, 843 (2010).

[16] T. A. Lozinskaya et al. Astron. Lett., 26, 77 (2000)

[17] Y. Uchiyama et al. Astrophys. J., 571, 866 (2002).

[18] V.G. Sinitsyna, V.Y. Sinitsyna Bull. of the Lebedev Phys. Inst., 40(5), 113 (2013).

[19] J. Lande et al. Astrophys. J., 756(1), 5 (2012).

[20] D. J. Thompson et al. Astrophys. J. Suppl., 101, 259 (1995).

[21] J. A. Esposito et al. Astrophys. J., 461, 820 (1996).

[22] A. W. Chen et al. Astron.\& Astrophys., 525, A33+ (2011).

[23] E. Aliu et al. 2013 Astrophys. J. 77093.

[24] A. A. Abdo et al. Astrophys. J., 734, 28 (2011).

[25] A. Weinstein ArXiv:0912.4492 